\title{
Physical Therapy Treatments Incorporating Equine Movement: A Pilot Study Exploring Kinetic Interactions between Children with Cerebral Palsy and the Horse
}

\author{
Priscilla Lightsey \\ Texas A and M University: Texas A\&M University College Station \\ Yonghee Lee
}

Texas A\&M University College Station

Nancy Krenek

Texas A\&M University College Station

Pilwon Hur ( $\square$ pilwonhur@tamu.edu )

Texas A\&M University College Station https://orcid.org/0000-0002-3651-1721

Research

Keywords: Hippotherapy, equine assisted therapy, interaction, children with cerebral palsy, functional mobility

Posted Date: April 5th, 2021

DOI: https://doi.org/10.21203/rs.3.rs-382424/v1

License: (c) (i) This work is licensed under a Creative Commons Attribution 4.0 International License. Read Full License 


\section{Abstract}

\section{Background}

Physical therapy treatments incorporating equine movement is recognized as an effective tool to treat functional mobility and balance in children with cerebral palsy (CP). To date, only a few studies examined kinematic outputs of the horses and children when mounted. In this pilot study, we examined the interaction between the horses and children with CP during physical therapy sessions where equine movement was utilized to better understand the effectiveness of this type of treatment.

\section{Methods}

Four children with CP participated in eight physical therapy sessions incorporating hippotherapy as a treatment intervention. Functional mobility was assessed using the Timed Up Go or 10m Walk Test. Inertial measurement unit sensors, attached to children and horses, recorded movements and tracked acceleration, angular velocity, and body orientation. Correlation between vertical accelerations of children and horses were analyzed. In addition, peak frequencies of vertical accelerations of children and horses were compared.

Results

Functional tests modestly improved over time. The children's movements, (quantified in frequency and temporal domains) increasingly synchronized to the vertical movement of the horse's walk, demonstrated by reduced frequency errors and increased correlation.

\section{Conclusions}

The findings suggest that as the sessions progressed, the participants appeared to become more familiar with the horse's movement. Since the horse's gait at a walk mimics the human gait this type of treatment may provide individuals with $\mathrm{CP}$, who have abnormal gait patterns, an opportunity for the neuromuscular system to experience a typical gait pattern. The horse's movement at the walk are consistent, cyclical, rhythmical, reciprocal and multi-dimensional which can facilitate motor learning. Thus, the increased synchronization between horse and the mounted participant suggests that physical therapy utilizing equine movement is a viable treatment tool to enhance functional mobility. This study may provide a useful baseline for future work.

Trial registration

Texas A\&M University Institutional Review Board. IRB2018-0064. Registered 8 March 2018. Link: https://rcb.tamu.edu/humans/irb and https://github.com/pilwonhur/HPOT

\section{Full Text}


Due to technical limitations, full-text HTML conversion of this manuscript could not be completed. However, the manuscript can be downloaded and accessed as a PDF.

\section{Figures}
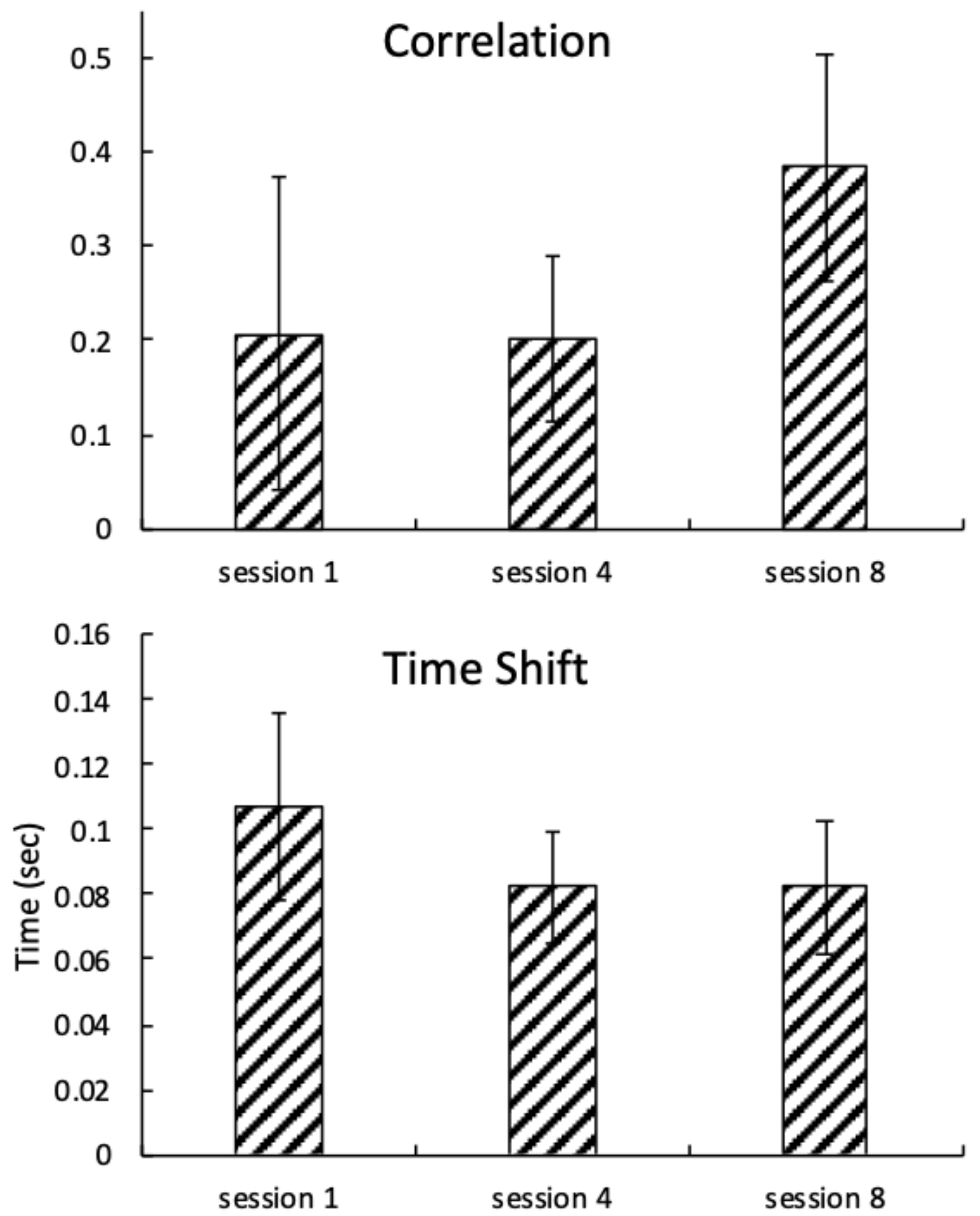

Figure 1 
Experimental protocol. Functional mobility tests were performed before and after the HPOT sessions. Each 20-min HPOT session consisted of 10-min continuous riding and 10-min riding with multiple gostops. The gure-of-eight patterns were made during the HPOT session.

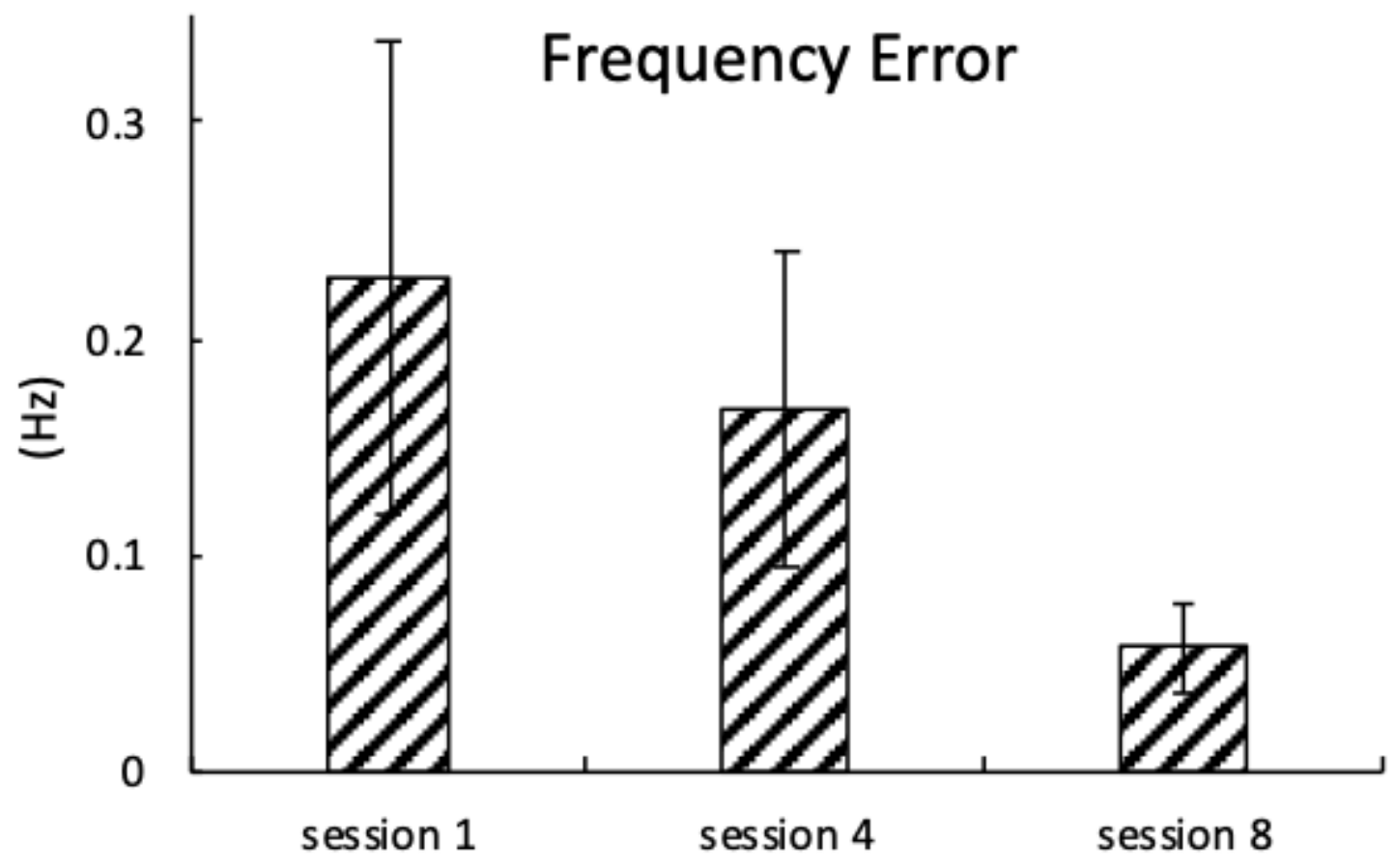

Figure 2

IMU sensors to capture the sinusoidal wave pattern of the horse's gait at a walk [18] and to examine how the rider and the horse interact. 

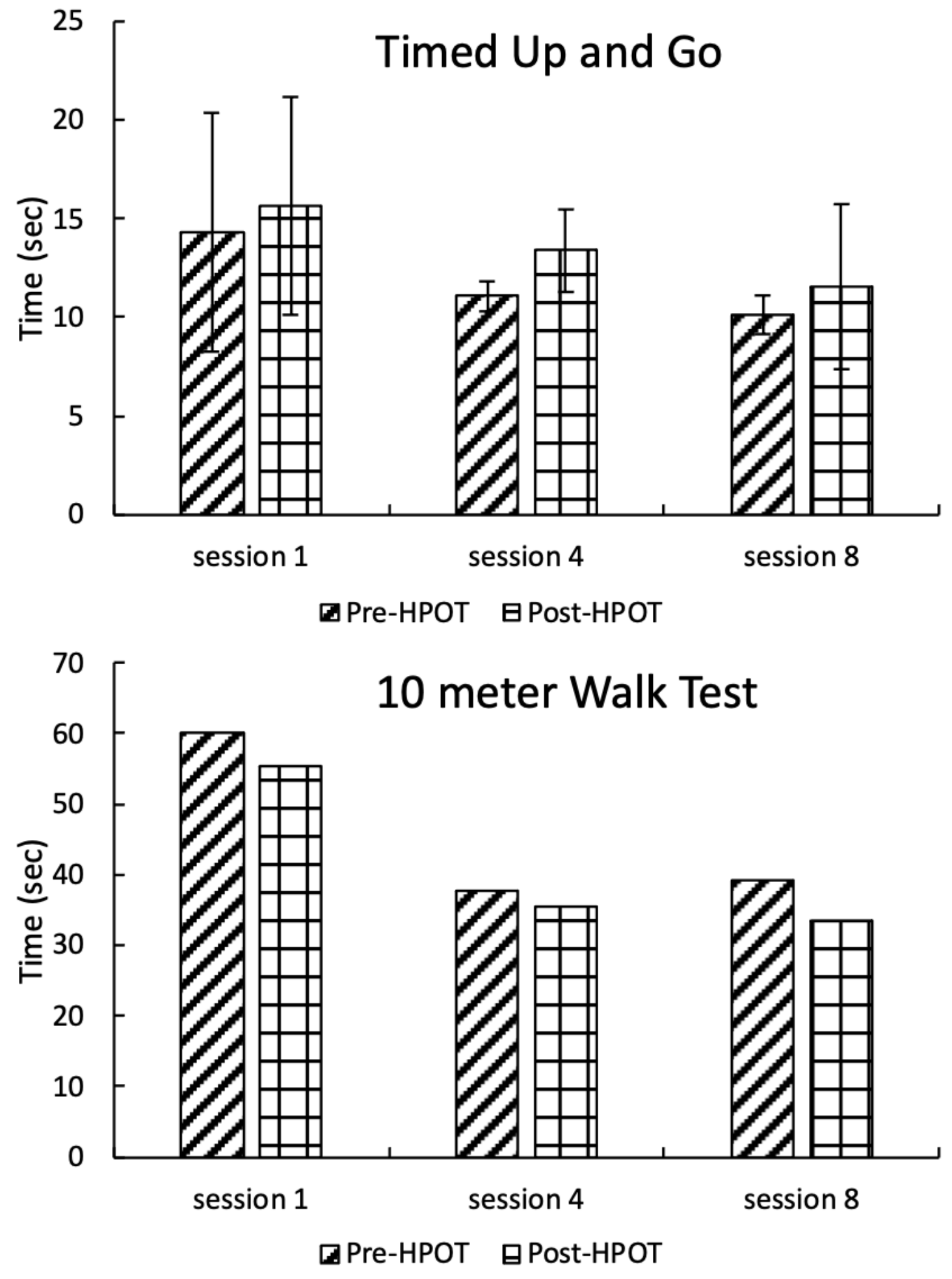

Figure 3

Sample plots of ACCz for both rider's head (i.e., IMU1 from Fig. 2) and horse's back (i.e., IMU2 from Fig. 2). ACC $z$ from IMU1 (in blue) lags ACCz from IMU2 (in red). 

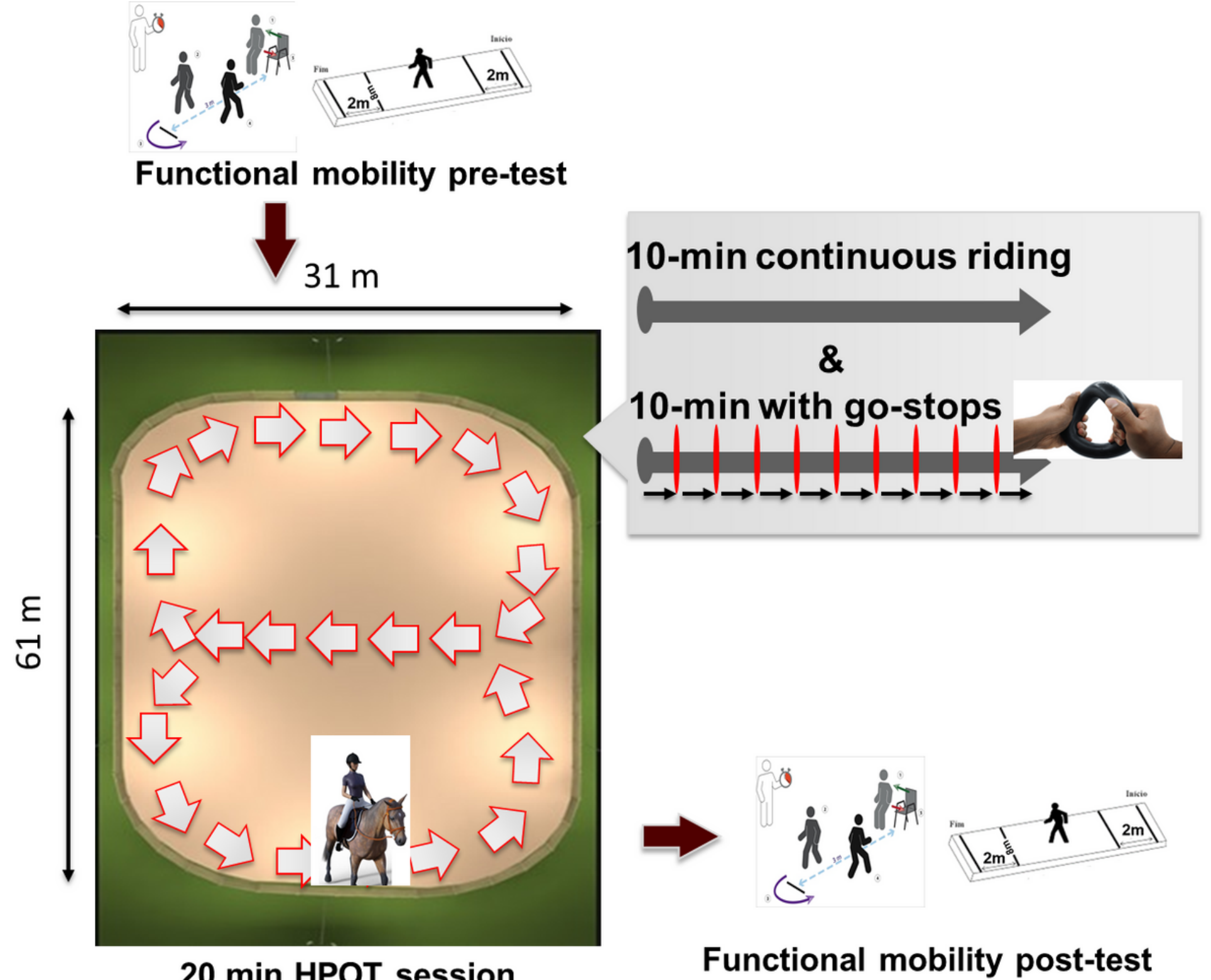

Functional mobility post-test

Figure 5

Bar graphs of the functional mobility tests. Top graph shows TUG results for participants 1-3 whereas bottom graph shows 10mWT for participant 4. Error bars in the top graph indicate one standard deviation. Bottom graph does not have the error bars since it involves with only one participant. 


\section{IMU sensors}
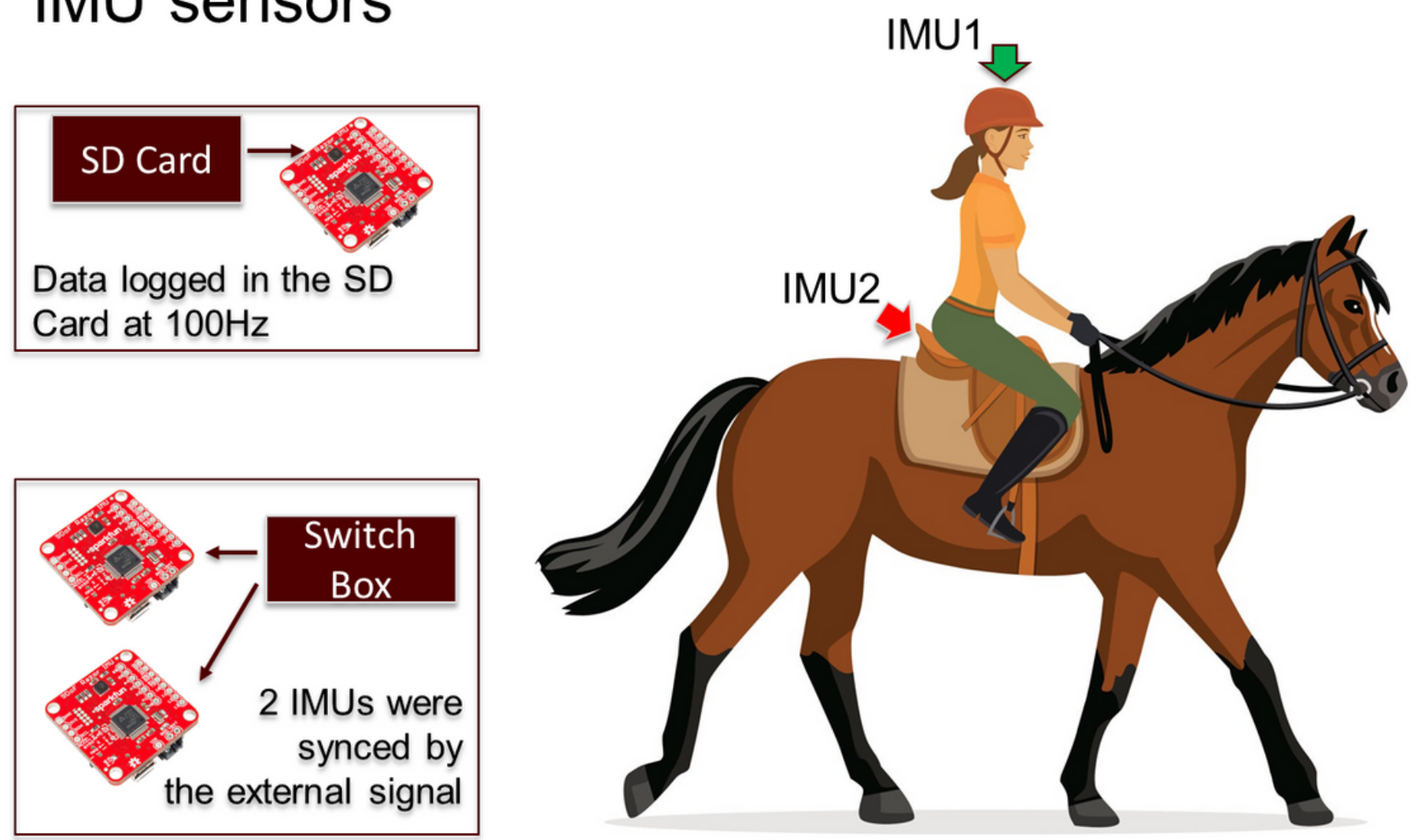

Figure 7

Root Mean Square Error (RMSE) between the peak harmonics of head ACCz and horse's back ACCz. Error bars indicate one standard deviation. 